\title{
Computer Simulation of 3-Dimensional Dendrite Growth Using Modified Phase Field Method
}

\author{
D. P. ZHAO, T. JING and B. C. LIU \\ Department of Mechanical Engineering, Tsinghua University, Beijing, P. R. China. E-mail: jingtao@mail.tsinghua.edu.cn \\ (Received on November 11, 2002; accepted in final form on January 15, 2003)
}

\begin{abstract}
Numerical simulation of three-dimensional dendritic growth is studied by using a macro-micro coupled method and a capturing liquid method. The dendritic growth is controlled by the solution of the phase field equation. Since it is difficult to compute the microstructure of a whole sample, a scheme is adopted that the temperature field is calculated on the whole sample, while the microstructure computation is carried out by selecting a macro-cell in the casting. Under the condition of not changing the Phase-field Model, a calculating method is advanced, which captures the liquid cells into the interface cells. In that method, every calculated micro-cell is endowed with a variable which identifies whether the cell is in the interface region and the phase field equation is only solved for the cells in that region. When the dendrite grows, the method captures the liquid cells into the interface ones and pushes the interface region forward. For the captured liquid cells, the values of the phase variable are modified. We apply the calculating method to accelerate the calculating speed, and realize the simulation of three-dimensional single grain and multiple grains for aluminum alloy. The simulation results are compared with those obtained experimentally.
\end{abstract}

KEY WORDS: phase field; capturing liquid; microstructure; dendrite.

\section{Introduction}

During the last decade, ${ }^{1-5)}$ the phase field models has been known as one of the most adequate deterministic models for directly simulating the dendrite growth morphology. Generally, it is considered that there are two approaches to derive the phase-field equation ${ }^{6}$ : the free energy method and the entropy method. The phase field method is based on Ginzburg-Landau theory, and reflects the influence of diffusion, ordering potential and thermodynamic driving force by using the differential equations. In the phase-field model, the state of the phase is represented continuously by an order parameter, the phase-field, $\phi$. For example, $\phi=1$, $\phi=0$ and a finite region in which $0<\phi<1$ represent solid, liquid and the interface, respectively. The time change of the phase-field is assumed to be proportional to variation of the free energy functional or variation of the entropy functional. By coupling the phase field to the temperature field, solute field, fluid field and other external fields, it offers the prospect of being able to simulate the realistic solidification processing. Besides, the phase field method does not need to track the solid/liquid interface, and therefore is very suitable for simulating the grain growth, especially for the $3 \mathrm{D}$ microstructure evolution. However, the study to simulate the microstructure of real castings is seldom performed. The reason may be in the two aspects: 1) first, it is difficult to compute the microstructure of the whole sample casting. Because that costs too long calculating time and demands insufferable computational memory, and the actual computer devices are unable to complete the overload computa- tional tasks; 2) second, the computational efficiency for solving the phase-field equation is too low. If the two problems were not solved, it would not come true to simulate the microstructure of real castings.

In this paper, we attempt to ravel out the above problems and realize the microstructure simulation of casting sample. A macro-micro coupled method is applied to avoid partitioning the whole casting sample beyond micro-scale. The temperature field is calculated for the whole casting, while the computation of the microstructure is only carried out by selecting a macro-cell in the central region of the casting, the macro temperature and the micro temperature are coupled. A calculating method, which captures liquid cells into interface ones, is advanced to improve the efficiency of solving the phase-field control equation. By the two calculating methods, we carry out the simulation for a single grain growth and multiple grains growth corresponding to two kinds of Al Alloys, and the simulation results are compared with those obtained experimentally.

\section{Mathematic Model}

\subsection{Phase-field Model}

The three-dimensional phase-field model is derived directly from the model advanced by Karma and Rappel. ${ }^{7)}$ The temperature field equation is given by

$$
u=\Delta T /\left(L / C_{\mathrm{p}}\right)
$$




$$
\partial_{t} u=D \nabla^{2} u+\frac{1}{2} \partial_{1} \psi
$$

where $u$ represent dimensionless temperature, $T_{\mathrm{M}}$ is the melting temperature, $L$ is the latent heat of the thermal diffusivity, $C_{\mathrm{p}}$ is the specific heat at constant pressure.

The phase-field control equation is given as follow

$$
\begin{aligned}
\tau(\vec{n}) \partial_{t} \psi= & {\left[\psi-\lambda u\left(1-\psi^{2}\right)\right]\left(1-\psi^{2}\right)+\vec{\nabla} \cdot\left[W(\vec{n})^{2} \vec{\nabla} \psi\right] } \\
& +\sum_{p} \partial_{p}\left[|\vec{\nabla} \psi|^{2} W(\vec{n}) \frac{\partial(W(\vec{n}))}{\partial\left(\partial_{p} \psi\right)}\right] \ldots \ldots \ldots \ldots . . .
\end{aligned}
$$

where $\psi$ is phase-field variable, represents the state of matter, $\psi=1, \psi=-1$ and a finite region in which $-1<\psi<1$ represent solid, liquid and the interface, respectively. $p=x$, $y, z$, represents the coordinates. The positive constant $\lambda$ controls the coupling between the temperature field and the phase field. $\tau$ is a relaxation time, $W$ is the interface thickness, both $\tau$ and $W$ are functions of the interfacial normal $\vec{n}$ in order to account for anisotropic surface energy and kinetics. $\tau$ and $W$ are assumed to take the form:

$$
\begin{aligned}
& W(\vec{n})=W_{0} A_{\mathrm{s}}(\vec{n}), \tau(\vec{n})=\tau_{0} A_{\mathrm{s}}(\vec{n})^{2}, \text { with } \\
& A_{\mathrm{s}}(\vec{n})=\left(1-3 \varepsilon_{4}\right)+4 \varepsilon_{4} \frac{\left(\partial_{x} \psi\right)^{4}+\left(\partial_{y} \psi\right)^{4}+\left(\partial_{z} \psi\right)^{4}}{|\vec{\nabla} \psi|^{4}} \ldots
\end{aligned}
$$

The phase-field model for multiple grains growth should adopt idealistically multi-phase model, such as the model advanced by Tiaden, ${ }^{8}$ the control equation is written as follow

$$
F(\phi, \nabla \phi)=\int_{V} \sum_{i=1}^{n} \sum_{j=1}^{i}\left(f_{i j}^{\mathrm{grad}}(\phi, \nabla \phi)+f_{i j}^{\mathrm{pot}}(\phi)\right) d V
$$

However, the multi-phase model has an evident flaw that more variables are required, that means demanding more memory and more calculating time. To cut down the computational task, we apply the same phase-field model to simulate the single grain growth and the multiple grains growth. It is assumed that all the grains share a phase-field variable and they have the same anisotropic characteristic. It is supposed to indicate that when the grains collide each other, the multi-phase model is more exact. Anyway, the factor can be ignored in our simulation.

\subsection{Solute Diffusion Model}

The mixed solute diffusion equation, derived by Beckermann et al., ${ }^{9}$ is used to describe the solute distribution.

$$
\frac{\partial}{\partial t}\left[(1+\psi+(1-\psi) k) C_{1}\right]=\nabla \cdot\left[\begin{array}{l}
(1-\psi) D_{\mathrm{s}} k \nabla C_{1} \\
+(1+\psi) D_{1} \nabla C_{1}
\end{array}\right]
$$

where $C_{1}$ is the concentration in liquid, $k$ is the partition coefficient, $D_{\mathrm{s}}$ is the solute diffusion coefficient in solid, $D_{1}$ is the diffusion coefficient of solute in liquid.

It is assumed that the solid and liquid composition at the solid/liquid interface is in equilibrium and there is no diffusion in solid. The solute partition between liquid and solid is given by: $C_{\mathrm{s}}=k C_{\mathrm{l}}$, where $C_{\mathrm{s}}$ is the solid concentration.

\subsection{Nucleation Model}

On the base of the macro-micro coupled method, the whole region for simulating microstructure evolution are also treated with a macro-cell. The amount of nucleuses is dependent on the undercooling of the macro-cell. When the amount of nucleuses increases, a nucleus is settled in a random position in the microstructure region. The Continuous Nucleation Model ${ }^{10)}$ is adopted, the variation of nucleating density, $d n / d\left(\Delta T^{\prime}\right)$, satisfies the Gaussin distribution.

$$
\frac{d n}{d\left(\Delta T^{\prime}\right)}=\frac{n_{\max }}{\sqrt{2 \pi} \Delta T_{\sigma}} \exp \left(-\frac{\left(\Delta T^{\prime}-\Delta T_{\max }\right)^{2}}{2 \Delta T_{\sigma}^{2}}\right) \ldots \ldots(7)
$$

where $\Delta T_{\max }$ is the maximal nucleating undercooling, $\Delta T_{\sigma}$ is standard deviation of undercooling. $n_{\max }$ is maximal nucleating density.

\section{Numerical Method}

\subsection{A Macro-Micro Coupled Method}

Since it is difficult to compute the microstructure of the whole sample casting, the temperature field of the casting is calculated, while the computation of the microstructure is only carried out by selecting a cell in the central region of the casting, and due to a larger ratio of length to radius and the symmetry, the calculation of the microstructure of the selected cell is predigested to two scale. Two different meshes and time steps are used, the larger mesh and time step $\Delta t$ are used for the calculation of the macroscopic temperature field, and the smaller mesh and micro time step $\delta t$ are adopted to compute the microstructure in the time step $\Delta t$ when the temperature of the selected cell falls down under the liquid temperature. Macro and micro computation are carried out alternately and the initial distribution of the temperature in the micro domain is obtained by interpolating the temperature of the cells near the selected cell, the interpolating can be written as

$$
T_{\text {micro }}=\sum_{i}^{n} l_{i}^{-1} T_{i} / \sum_{i}^{n} l_{i}^{-1}
$$

where $l_{i}$ represents the distance between the micro-cell and the macro-cell, $i$.

\subsection{A Calculating Method Which Captures Liquid Cells into Interface Ones}

Here, a calculating method, which captures liquid cells into interface ones, is advanced to improve the computing efficiency. In that method, every calculated cell is endowed with a variable, $S(i, j, k): S=-1$ identifies that the cell is in the liquid state $(\psi=-1) ; S=0$ identifies that the cell is in the interface region $(-1<\psi<1) ; S=1$ identifies that the cell is in the solid state $(\psi=1)$. During the simulating process, the phase field variable is only solved for the cells 
in the interface region and the remelting of solid cells is ignored. When the dendrite grows, the method captures the liquid cells into the interface ones and pushes the interface region forward. The rule which capturing liquid cells can be summarized as:

When some cell, $U(i, j, k)$, became solid state, we scan those cells near that cell $\left(r<=R_{\mathrm{s}}\right)$ to decide whether they are in the liquid state $(S=-1)$, if they are, then set $S=0$, turning them into interface cells, and modify their phasefield value by setting $\psi=P(r)$.

In the above statement, $U(i, j, k)$ is any micro-cell, $r$ is the distance between some cell and the just solidified cell $U(i, j, k), R_{\mathrm{s}}$ is the interface thickness, $P(r)$ is a function which may have different forms. In this paper, $P(r)$ is given referring to the order parameter solution in one-dimensional space by Collins. ${ }^{11)}$ The solution is obtained under the diffuse interface model and assuming steady-state conditions. $P(r)$ should also be the function of undercooling, here we simplify the Collins' solution into the following form

$$
P(r)=1-a \cdot \tanh \left(r / R_{\mathrm{s}}\right) \text {. }
$$

where $a$ is constant, the scanning radius $r \in\left[0, R_{\mathrm{s}}\right], P(r) \in$ $[-1,1]$.

It is worth discussing the validity and correctness of the calculating method. Because this method solved the phasefield equation only in the interface region, it reduces greatly the computing total time and improves the computing efficiency. By modifying the phase-field value of the captured liquid cells, the continuity of interface can be ensured and it is avoided that the interface thickness decreases so that the interface region vanishes. However it needs to discuss whether the modification is reasonable. In the early time, the interface-tracking model considered the interface thickness as zero, in the sequel, the phase-field model assumed that a interface region existed, actually, it is not very important whether there is a real interface region between liquid and solid. The hypothesis that an interface region exists in the phase-field model also allows modifying reasonably the interface. On the other hand, the modification does not offend the energy conversation theory. In conclusion, the capturing liquid method is feasible.

Another problem is whether it is possible that the phasefield value varies rapidly in the space when the phase-field value of those just captured cells. If setting $\psi=P(r)$ arose smart variation, the calculating method would be in failure. When any cell solidifies, the very close cells to that cell must have been in non-liquid state, the captured cells' position, $r$, also is close to $R_{\mathrm{s}}$, so $P(r)$ is near -1 and no smart phase-field variation brings out. The stabilization of the computing result also verifies that point.

\section{Results and Discussion}

\subsection{A Single Grain Growth}

In order to simulate the three-dimensional equiaxed dendritic growth under the realistic condition, a casting sample of $\mathrm{Al}-4 \mathrm{wt} \% \mathrm{Cu}$ alloy was poured with pouring temperature at $710^{\circ} \mathrm{C}$. The thermophysical properties of the alloy is given by Diepers, ${ }^{12)}$ and all the values of parameters used in the calculation are given in Table 1. It is necessary to indicate that the values of $W_{0}, \tau_{0}$ and $\varepsilon_{4}$ are dimensionless. The computational microstructure domain in the center of the casting is divided with a cell size of $2 \mu \mathrm{m}$, while the macro grid size is $0.5 \mathrm{~mm}$. The nucleus is assigned in the center of the domain. The computational time is about $60 \mathrm{~h}$ under a CPU-1.7 GHz computer.

The simulated process of a single grain growth is shown in Fig. 1. From Figs. 1(a)-1(d), it can be found that the anisotropy of dendrite growth was more and more obvious. From Fig. 1(b) on, neck-shrinking occurs at the root of the main branch. In the Fig. 1(d), obvious neck-shrinking phenomenon appears on the section of the main branch. Neckshrinking is a reasonable result of the anisotropic interface. It is cheering that the side branches begin to appear in Fig. 1(b), the side braches are very important to predict the material mechanical properties. The development of side branches is considered as the result of noise, such as thermal noise, concentration noise, phase-field value noise and other noises. In the field-model, there is two kind of noise incorporated into the model: the conserved noise in the heat current and the nonconserved noise in the evolution equa-

Table 1. The numerical values of the parameters used in the calculation.

\begin{tabular}{ccc}
\hline Property & Value(Al-4wt.\%Cu) & Value $(\mathrm{Al}-7 \mathrm{wt} . \% \mathrm{Si})$ \\
\hline$W_{0}$ & 1 & 1 \\
$\tau_{0}$ & 1 & 1 \\
$\varepsilon_{4}$ & 0.03 & 0.03 \\
$T_{m}$ & $933.6 \mathrm{~K}$ & $934 \mathrm{~K}$ \\
$m_{l}$ & $2.6 \mathrm{~K} / \%$ & $7.7 \mathrm{~K} / \%$ \\
$C_{p}$ & $2.58 \times 10^{6} \mathrm{~J} / \mathrm{K} \mathrm{m}^{3}$ & $2.35 \times 10^{6} \mathrm{~J} / \mathrm{K} \mathrm{m}^{3}$ \\
$L$ & $9.5 \times 10^{8} \mathrm{~J} / \mathrm{m}^{3}$ & $9.5 \times 10^{8} \mathrm{~J} / \mathrm{m}^{3}$ \\
$D_{l}$ & $3 \times 10^{-9} \mathrm{~m}^{2} / \mathrm{s}$ & $3 \times 10^{-9} \mathrm{~m}^{2} / \mathrm{s}$ \\
$D_{s}$ & $3 \times 10^{-13} \mathrm{~m}^{2} / \mathrm{s}$ & $3 \times 10^{-13} \mathrm{~m}^{2} / \mathrm{s}$ \\
$k$ & 0.14 & 0.117 \\
$\Delta T_{\text {max }}$ & & $4.5 \mathrm{~K}$ \\
$\Delta T_{\sigma}$ & & $0.5 \mathrm{~K}$ \\
$n_{\max }$ & & $3.2 \times 10^{12} / \mathrm{m}^{3}$ \\
\hline
\end{tabular}

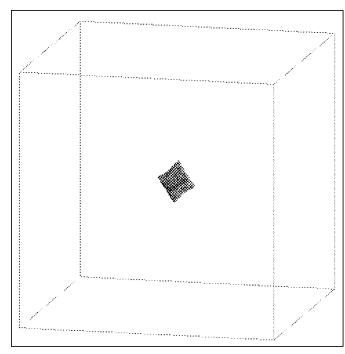

(a) $\mathrm{t}=0.12 \mathrm{~s}$

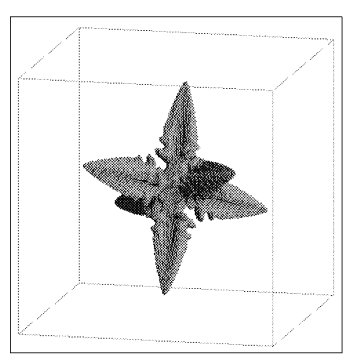

(c) $\mathrm{t}=0.7 \mathrm{~s}$

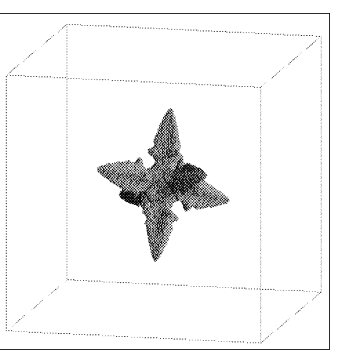

(b) $\mathrm{t}=0.42 \mathrm{~s}$

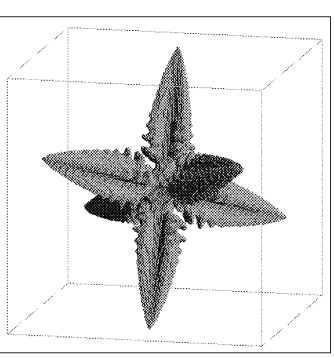

(d) $\mathrm{t}=0.8 \mathrm{~s}$
Fig. 1. The dendrite morphology at different time. (a) $t=0.12 \mathrm{~s}$, (b) $t=0.42 \mathrm{~s}$, (c) $t=0.7 \mathrm{~s}$, (d) $t=0.8 \mathrm{~s}$. 
tion for the phase-field variable. ${ }^{13)}$ By Karma ${ }^{13)}$ and Tong, ${ }^{14)}$ the conserved noise is more relevant with dendrite sidebranching. However, it is unsure whether the conserved noise is the absolute reason of the appearance of side branches. For example, there were obvious side branches in the interface-tracking model, ${ }^{15)}$ but no noise entered into the control equation. In our model, no noise is incorporated.

Figure 2 shows the profile of the tip growth diameter of the dendrite about time. At the beginning, because the interface is set freely, the tip diameter is unstable; when the grain grows stably, the tip diameter becomes stable. The simulating results were compared with those obtained experimentally in order to test the simulations. Figure 3 is microstructure morphology obtained from the casting sample. In respect that the nucleating position is random and the position relation between the sampling plane and the grain is very difficult to decide, it would be impossible that compare exactly the realistic microstructure morphologies and the simulating ones. But their morphologies can be compared from the integral shape, from Figs. 1 and 3, the same characteristics can be found: dendrite, the anisotropic growth and side-branching.

The width of the main branch was also compared quantitatively as well as the secondary dendrite arm spacing (SDAS). The simulating width of the main branch can be represented roughly by the tip diameter, from Fig. 2, the width range is $30-35 \mu \mathrm{m}$; Fig. 3 shows that the sampling width is $20-40 \mu \mathrm{m}$. From Fig. 1(d), SDAS is $30-40 \mu \mathrm{m}$, the

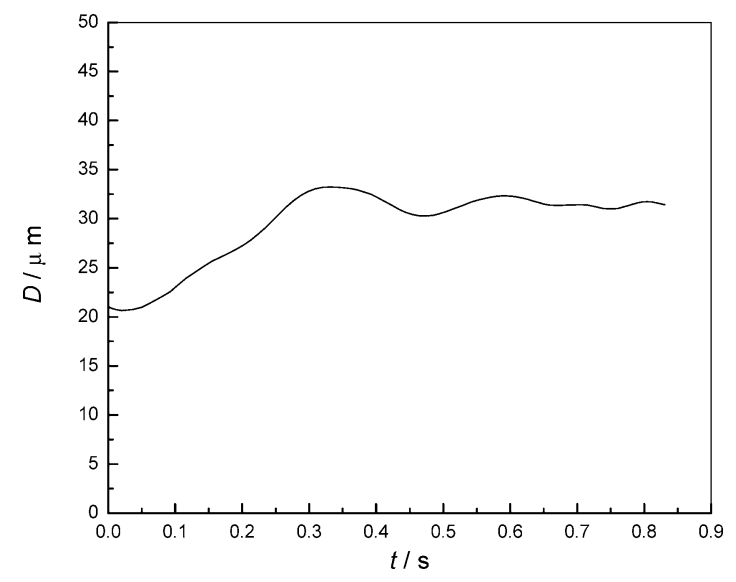

Fig. 2. The tip growth diameter of one main branch.

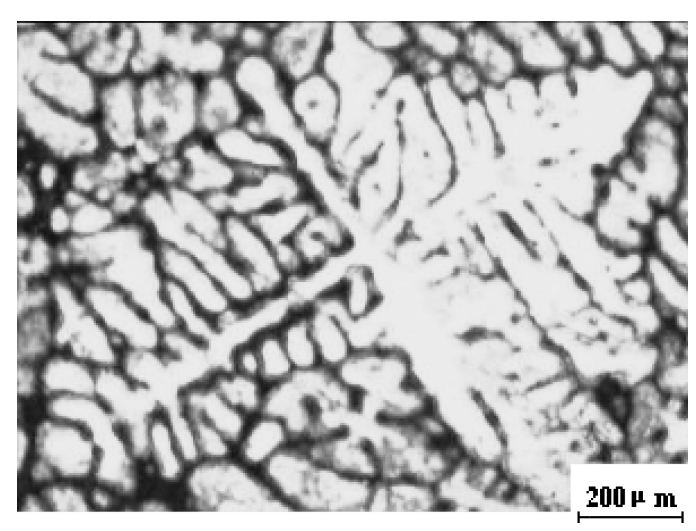

Fig. 3. The microstructure of the Al-Cu alloy sample. sampling SDAS is $35-45 \mu \mathrm{m}$ from Fig. 3. It can be concluded that the two results are consistent. The comparison verifies the calculating is correct and valid.

\subsection{Multiple Grains Growth}

A casting sample of $\mathrm{Al}-7 \mathrm{wt} \% \mathrm{Si}$ alloy was poured with pouring temperature at $720^{\circ} \mathrm{C}$ in order to simulate the multiple grains growth. The values of parameters used in the calculation are given in Table 1. The sample is divided into macro grids with a grid size of $1 \mathrm{~mm}$, while the microstructure region is divided with a cell size of $2 \mu \mathrm{m}$. The computational time is about $430 \mathrm{~h}$ under a CPU-266 MHz workstation.

Figure 4 shows the process of the multiple grains growth, the nucleating position is seldom, but the anisotropic characteristic of each grain is considered to be same. From Fig. 4(c) on, the nucleation is completed. Figure $\mathbf{5}$ is sampling microstructure morphology, some grains' morphologies are not integral, but we can compare the grain density roughly between the simulation result and

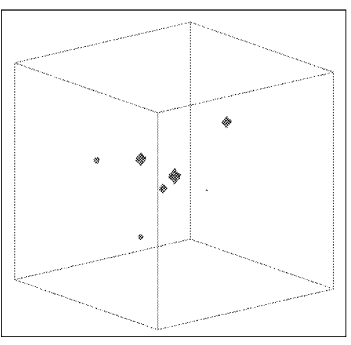

(a) $\mathrm{t}=0.05 \mathrm{~s}$

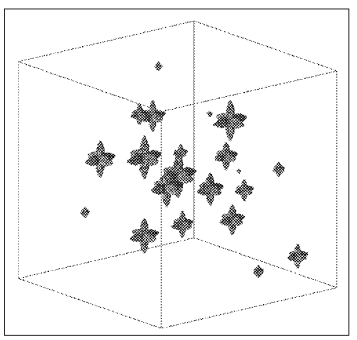

(c) $\mathrm{t}=0.3 \mathrm{~s}$

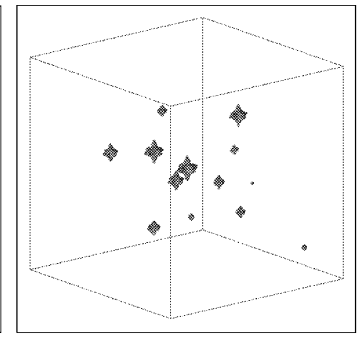

(b) $\mathrm{t}=0.1 \mathrm{~s}$

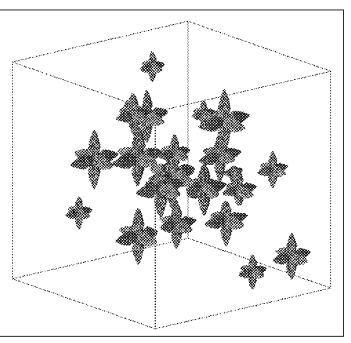

(d) $\mathrm{t}=0.4 \mathrm{~s}$
Fig. 4. The morphologies and distribution of grains with different time. (a) $t=0.05 \mathrm{~s}$, (b) $t=0.1 \mathrm{~s}$, (b) $t=0.3 \mathrm{~s}$, (d) $t=$ $0.4 \mathrm{~s}$.

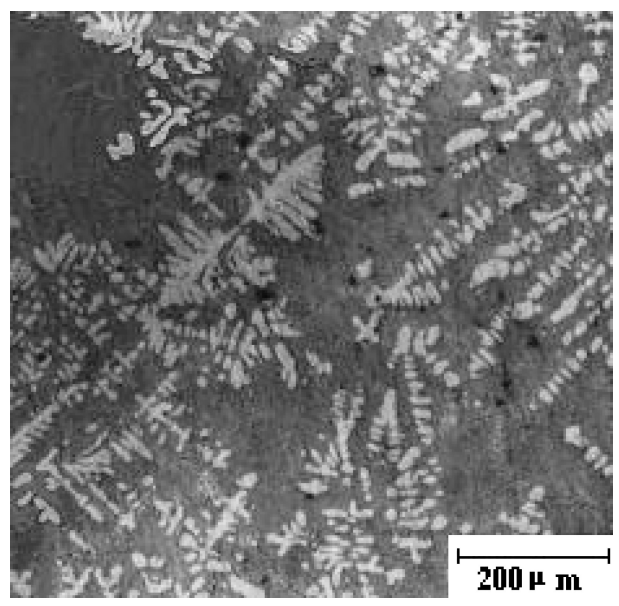

Fig. 5. The microstructure of the Al-Si alloy sample. 


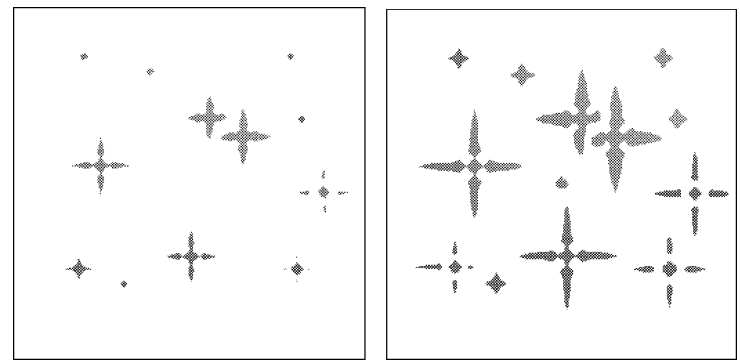

Fig. 6. Sections through the center of the simulated cubic mesh at one time.

the sampling result. If we define the plane grain density, $N_{\mathrm{S}}$, as the grain number per area units and the volume grain density, $N_{\mathrm{V}}$, as the grain number per volume units, the relation between $N_{\mathrm{S}}$ and $N_{\mathrm{V}}$ can be derived roughly as

$$
N_{\mathrm{S}}=\sqrt[3]{N_{\mathrm{V}}^{2}}
$$

The sampling grain density is 71 grains per $\mathrm{mm}^{2}$, the simulating result is 492 grains per $\mathrm{mm}^{3}$, equal to 62 grains per $\mathrm{mm}^{2}$. In some degree, the two results are consistent.

Figure 6 is two sections through the simulated cubic mesh. Ideally, Fig. 6 should fit the Fig. 5, but in our simulating results, they did not fit well. However, at the present, it is almost impossible to achieve that kind of simulation result. One side, Fig. 5 is not the final growth morphology, but Fig. 6 shows the solidified ultimately morphology. On the other hand, the mathematic models did not include convection and the true reason of side branching is not clear. However, an intriguing phenomenon, the competition growth was found. From Fig. 6, we can find that the lengths of some grains are not equal at the four main direction. It can be explained as: when the main branches of two different grains grow toward each other, due to the growth competition exists, one main branch restrains the growth of the other branch each other, at last, their growth both are retarded. A conclude can be arrived that the position relation between grains can affect their growth.

\section{Concluding Remarks}

In this paper, we attempted to simulate the dendrite growth for the casting samples. In order to avoid dividing the whole sample with the micro-scale, the macro-micro coupled method was advanced, that method decrease greatly the computing task and make the simulation more feasible. Another calculating method, which captures the liquid cells into interface ones and modifies the phase-field value, was developed to improve the efficiency of solving the phase-field equation. By using the two computing method, it was realized that the simulation of a single grain growth and multiple grains growth in the real samples. The simulation results were agreed with those obtained experimentally, it proved that the two methods was valid and correct.

\section{Acknowledgment}

This work was supported by the National Science Foundation of China (No. 50005011)

\section{REFERENCES}

1) R. Kobayashi: Physica D, 63 (1993), 410.

2) A. A. Wheeler, B. T. Murray and R. J. Schaefer: Physica D, 66 (1993), 243.

3) A. Karma and W. J. Rappel: Phys. Rev. Lett., 77 (1996), 4050.

4) Q. Li and C. Beckermann: Phys. Rev. E, 57 (1998), 3176

5) S. G. Kim, W. T. Kim, J. S. Lee, M. Ode and T. Suzuki: ISIJ Int., 39 (1999), 335.

6) M. Ode, S. G. Kim and T. Suzuki: ISIJ Int., 41 (2001), 1076.

7) A. Karma and W. J. Rappel: Phys. Rev. E, 57 (1998), 4323.

8) J. Tiaden, B. Nestler, H. J. Diepers and I. Steinbach: Physica D, 115 (1998), 73

9) C. Beckermann, H. J. Diepers and I. Steinbach: J. Comput. Phys., 154 (1999), 468.

10) P. Thévoz, J. L. Kesbiollex and M. Rappaz: Metall. Trans., 20A (1989), 314

11) J. B. Collins and H. Levine: Phys. Rev. B, 31 (1985), 6119

12) H. J. Diepers, C. Beckermann and I. Steinbach: Acta Mater., 47 (1999), 3663.

13) A. Karma and W. J. Rappel: Phys. Rev. E, 60 (1999), 3614.

14) X. Tong, C. Beckermann, A. Karma and Q. Li: Phys. Rev. E, 63 (2001), 061601.

15) R. Almgern: J. Comput. Phys., 106 (1993), 337. 